\title{
Fallbeispiele zur Anwendung von Heilpilzen in der Tiermedizin
}

\author{
Svenja Thiede
}

\section{Zusammenfassung}

Im vorliegenden Artikel wird die Mykotherapie als Möglichkeit eines individuellen, ganzheitlichen Therapieansatzes für Tiere vorgestellt. Die verwendeten Heilpilze und ihre Wirkungsweise werden kurz vorgestellt und Anwendungsbeispiele gegeben.

\section{Heilpilze}

Die Anwendung von Ständerpilzen in der Heilkunde hat eine Jahrtausende alte Geschichte und ist Teil der Traditionellen Chinesischen Medizin. Allerdings wurde vermutlich auch in Europa bereits in der Frühzeit mit Heilpilzen gearbeitet, denn bei „Ötzi“, der vor 5200 Jahren unterwegs war, wurde in seiner Reiseapotheke ein Verwandter des Coriolus versicolor gefunden [4].

Bei den in der Heilkunde verwendeten Pilzen handelt es sich um Ständerpilze, die zu den Speisepilzen gehören. Ständerpilze bestehen aus dem oberirdisch wachsenden Fruchtkörper, in der Regel Hut und Stiel in charakteristischer Form, dem Mycel in den oberen Schichten des Nährbodens und den feinen Hyphen, die den gesamten Boden durchziehen. Der Fruchtkörper dient der Produktion der Sporen zur Verbreitung des Pilzes, während Mycel und Hyphen der Nährstoffversorgung aus dem Boden dienen.

\section{Struktur und Funktion}

Die Pilze durchlaufen unterschiedliche Wachstumsphasen. Sie „schießen“ aus dem Boden, wenn das Mycel seine Inhaltsstoffe in Form der Fruchtkörper entleert. Aufgrund der Wachstumsphasen sind im Fruchtkörper andere Nährstoffe enthalten als in Mycel, Hyphen oder Sporen. Deshalb wird empfohlen, in der Heilkunde immer Präparate aus dem ganzen Pilz mit all seinen Anteilen zu verwenden, um alle positiven Eigenschaften des Pilzes zu nutzen [4].

Im ökologischen Kreislauf haben Pilze die Aufgabe, organische Materie zu zerlegen, um die Nährstoffe wieder verfügbar zu machen. Pilze speichern Schwermetalle und radioaktive Isotope im Fruchtkörper, was den Boden entlastet, aber den Verzehr von Wildpilzen problematisch macht. Sie können für Säugetiere toxische Substanzen aufspalten und somit unschädlich machen. 80-90\% aller Pflanzen leben in Symbiose mit Pilzen und werden durch diese in ihrem Wachstum gefördert und zum Teil durch Pilztoxine vor Feinden geschützt (z.B. Endophyten in Weidegräsern [8]).

\section{Inhaltsstoffe}

Wertgebende Inhaltsstoffe der heilkundlich verwendeten Speisepilze sind hohe Gehalte an Eiweiß, besonders Glykoproteine, Adenosin, Chitin und Selen. Sie enthalten Vitamine der B-Gruppe und Folsäure, Vitamin D bzw. Ergosterin sowie Vitamin E und auch diverse Enzyme.

Für die Qualität des verwendeten Pilzpräparates ist die Anzucht der Pilze, das verwendete Substrat, die schonende Ernte zum richtigen Zeitpunkt und sofortige Verarbeitung von entscheidender Bedeutung. Pilzpulver, welches alle Anteile des Pilzes enthält, wirkt ausgleichend und regulierend.

Die wichtigsten Inhaltsstoffe sind die Glykoproteine mit speziellen beta-Glukanketten. Diese Glykoproteine fördern die Leistungsfähigkeit des Immunsystems auf verschiedenen Ebenen. Kurz vor dem Abwurf der Sporen sind die Glykoproteine ausgereift, sodass der richtige Erntezeit- punkt entscheidend ist. Da die Proteine bei $60^{\circ} \mathrm{C}$ denaturieren, ist eine Trocknung und Vermahlung bei möglichst niedrigen Temperaturen wichtig, um Struktur und somit Wirksamkeit der Glykoproteine zu erhalten [2].

Die Glykoproteine der Pilze lösen Immunantworten aus, ähnlich wie Pathogene: Die Zytotoxizität von Makrophagen, neutrophilen Granulozyten und T-Lymphozyten wird erhöht, die Ausschüttung von Zytokinen gefördert, dendritische Zellen werden aktiviert und die Produktion von NO-Radikalen angeregt. Auf diese Weise wird die unspezifische Abwehr gegen Bakterien und Viren gefördert und die Reaktion gegen neoplastische Veränderungen verstärkt [2]. Diese Reaktionen werden unter anderem dadurch ausgelöst, dass die beta-Glukane Gemeinsamkeiten haben mit den Pathological Associated Molecular Pattern (PAMP) der pathogenen Mikroorganismen [2].

Beta-Glukane senken den Cholesterinund Triglyzeridspiegel. Der Glukosespiegel wird nach dem Essen gesenkt durch eine Erhöhung des Insulinspiegels und eine Sensibilisierung der Insulinrezeptoren. Dies führt zu einer schnelleren Verarbeitung von Glukose.

Die Pilze enthalten verschiedene Enzyme, die therapeutisch unterstützend wirken können, u.a. antioxidative Enzyme wie SOD (Superoxid-Dismutase), entgiftende Enzyme wie Cytochrome-Reduktasen und Peroxidasen und Enzyme, die eine überschießende Zellproliferation unterdrücken [2].

Adenosin verbessert die Fließeigenschaften des Blutes durch Senkung der Thrombozytenaggregation und durch Vasodilatation. Es hat außerdem eine bronchodilatatorische Wirkung. Neurotransmitter wie Acetylcholin, Noradrenalin, Dopamin und Koffein werden blockiert. 
Triterpene gehören zur Gruppe der Lipide, aus denen sich Steroide ableiten. Das in Pilzen enthaltene Ergosterin ist eine Vorstufe des Ergocalciferol.

Triterpene verbessern die Funktion der Nebennierenrinde. Ähnlich wie Cortisol sind sie in der Lage, die Histaminausschüttung bei Entzündungen und allergischen Reaktionen zu reduzieren.

Bei Bluthochdruck wird das Angiotensin converting Enzyme (ACE) gehemmt. Die Thrombozytenaggregation wird gehemmt. Es konnten u. a. zytotoxische Wirkungen und Hemmung der Phospholipase A nachgewiesen werden. Bestimmte Triterpene hemmen die reverse Transkriptase von HI-Viren [2].

\section{Adaptogene Wirkung}

Heilpilze sind sogenannte Adaptogene (N. Lazarev 1947 in [4]): Substanzen zur Aufrechterhaltung der Leistungsfähigkeit und Widerstandskraft des Körpers in Stresssituationen. Sie führen durch ihre unspezifische Wirkung zu einer Regulation verschiedenster Stoffwechselvorgänge und verbessern die Anpassungsfähigkeit des Makroorganismus durch einen normalisierenden Effekt, unabhängig davon, in welche Richtung die Abweichung stattgefunden hat. Als primäre Wirkung wird das Hypothalamus-Hypophysen-Nebennierenrinden-System und das Sympathoadrenerge System reguliert. Sekundär wirken Adaptogene auf Herz-Kreislauf, Leber, Pankreas und Nieren. Werden nur Extrakte der Pilze verwendet, so geht diese übergeordnete adaptogene Wirkung verloren.

\section{Anwendungsbeispiele \\ Equine Odontoclastic Tooth Resorp- tion and Hypercemetosis (EOTRH)}

Die EOTRH (Equine Odontoclastic Tooth Resorption and Hypercemetosis) des Pferdes wurde 2008 zum ersten Mal beschrieben [6]. Es handelt sich offenbar um eine multifaktorielle Erkrankung, die durch verschiedene Risikofaktoren begünstigt wird [1]. Zurzeit sind drei Formen der EOTRH beschrieben. Der resorptive Typ geht mit massiven Entzündungen der Zahnumgebung einher, der hyperzementotische Typ stellt sich ein, wenn die Entzündung abklingt und reparative Prozesse einsetzen, die schließlich zur Hyperzementose führen. Die dritte Form ist der Mischtyp aus beiden [3]. Sykora et al. [7] haben eine Beteiligung von Treponemen (Schraubenbakterien) nachgewiesen.

In der Praxis zeigt sich, dass die Zahnveränderungen in der Regel mit mehr oder weniger deutlichen Stoffwechselveränderungen oder -erkrankungen einhergehen. Viele Pferde mit EOTRH haben bereits ein nachgewiesenes Cushing-Syndrom, ein nachgewiesenes Equines metabolisches Syndrom (EMS) - oder deutliche Anzeichen für ein beginnendes EMS, Hufrehe in der Vergangenheit, Sommerekzem (Erfahrung aus eigenem Patientengut, mündl. Mitteilung von Zahnkollegen).

Bereits 2012 zeigten Simhofer und Koweika [5], dass Heilpilze eine positive Wirkung bei EOTRH haben können. Hier wurde eine Fertigmischung verwendet aus Cordyceps, Reishi, Pleurotus und Antrodia. Es handelt sich um eine „Pilzmyzelmischung zur Kräftigung und zum Schutz von Zahnfleisch und Zähnen“. Über Herkunft und Verarbeitung der Pilze dieser Mischung ist leider nichts bekannt, die Deklaration ist lückenhaft.

Da die EOTRH mit unterschiedlichen Veränderungen bzw. Begleiterkrankungen einhergeht, sollte auch die Mischung der Heilpilze individuell abgestimmt werden. In einer ausführlichen Anamnese muss die Gesamtsituation des Pferdes geklärt werden. Dabei spielt nicht nur die Stoffwechsellage eine Rolle, sondern auch die Grundfütterung. Heulage scheint negative Wirkung auf die Entwicklung der EOTRH zu haben (eigene Beobachtung, es liegen hierzu keine klinischen Daten vor).

Mit einer individuell ausgewählten Mischung von jeweils 2-4 Heilpilzen konnte das Fortschreiten der EOTRH bei verschiedenen Pferden gebremst werden.

Fall 1

Islandstute, geb. 1995, Erstanamnese Juli 2013. EOTRH und EMS waren bereits diagnostiziert. Zum Zeitpunkt der Anamnese Zahnfleisch mit Eiterfisteln über I1/I2 rechts, weitere nicht eiternde Fistel über I4, Zähne etwas gedreht, Palpation schmerzhaft, nicht gerötet, keine Einschränkungen beim Fressen, Allgemeinbefinden ungestört. Die behandelnde Pferdeklinik wollte I 1 und 12 bereits ziehen, weil die Zähne sich bereits lockerten. Die Besit- zerin hatte sich gemeldet, weil sie das Ziehen der Zähne gerne abwenden wollte.

Das Pferd bekam zu diesem Zeitpunkt ein Pilzpräparat mit 20\% einer Mischung aus fünf Pilzen, 40\% Hafer, 40\% Soja, täglich $10 \mathrm{~g}$. Enthalten waren laut Deklaration Agaricus blazei, White Beech, Brown Beech, Pleurotus eryngyii, Cordyceps militaris. Über Herkunft und Verarbeitung der Pilze gab es keine Information, das Präparat roch nach Getreide und war grob gemahlen.

Aufgrund der Anamnese wurde ein Pilzpulver aus reinen ganzen Pilzen der Firma Mycovital verordnet:

Reishi und Cordyceps, Hericium und Maitake, je zwei Pilze in einer Mischung in Kapseln.

Reishi: Zuckersenkung, Leberstoffwechsel

- Cordyceps: Cordycepin wirkt antibiotisch, stärkt die Knochen

- Hericium: Zahnfleisch, Schleimhaut

Maitake: Immunregulator, EMS

Erste Woche je eine Kapsel, ab der zweiten Woche je $2 \times 1$ Kapsel. Bei der Kontrolluntersuchung nach drei Wochen waren die Fisteln nicht mehr auffindbar, es bestand aber noch leichter Druckschmerz.

Bei der Kontrolle beim Zahnarzt im September gab es keine Veranlassung, die Incisivi zu ziehen, da diese fest saßen. Das Röntgenbild zeigte laut Bericht ggr. parodontale Entzündungen.

Aus Kostengründen wurde ab November die Gabe der Pilze unterbrochen. In der Folge zeigten sich bei der Zahnkontrolle im Januar wieder Eiterfisteln über I1/I2 oben rechts. Im weiteren Verlauf lockerten sich die Zähne und die Zahnwurzeln wurden zunehmend hyperzementotisch, sodass die beiden Incisivi im März 2014 gezogen wurden.

\section{Fall 2}

Ponystute, geb. 1997. Im November 2014 wurde EOTRH mit hochgradiger Gingivitis im Oberkiefer diagnostiziert. Das Pferd hatte zuvor Heulage bekommen. Es war häufig geringgradig tympanisch, geringgradig übergewichtig, das ganze Pony wirkte aufgeschwemmt. Nach der Anamnese wurde eine Mischung aus

- Polyporus: Aktivierung des Lymphflusses 


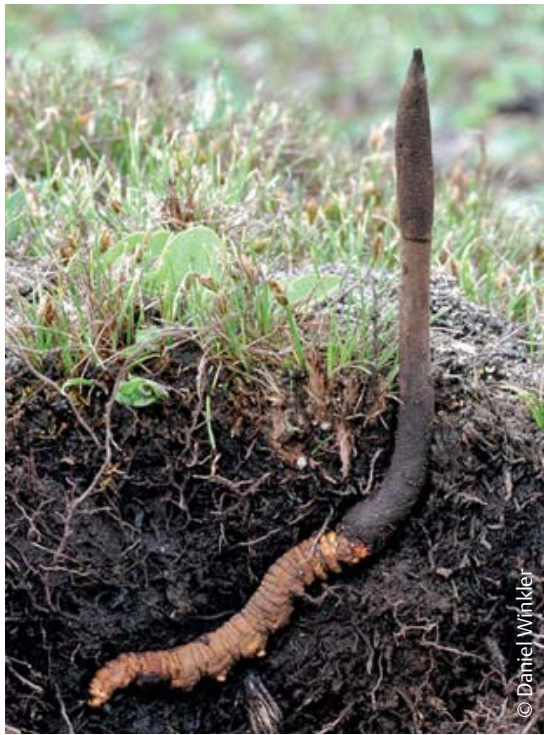

Abb. 1 Cordyceps mit Raupe.

Cordyceps: Cordycepin wirkt antibiotisch, stärkt die Knochen

- Hericium: Zahnfleisch, Schleimhaut von der Firma Mycovital empfohlen, welche ab November 2014 bis November 2016 verabreicht wurde. Dosierung: mit $1 / 2 \mathrm{Kap}-$ sel/Tag beginnen, langsam auf drei Kapseln/Tag steigern. Im Januar 2017 plötzlich hochgradige eitrige Gingivitis, ca. 3 Monate nach Absetzen der Heilpilze.

Es hat sich gezeigt, dass die Gabe an Pferde über Eimerfutter problemlos ist (inklusive der Kapsel). Die Pilze können offensichtlich den Entzündungsprozess bei der EOTRH bremsen oder reduzieren, können aber keine langfristige, endgültige Heilung bringen. Der Einsatz der Pilze muss also immer als Dauerbehandlung geplant werden und ist mit entsprechenden Kosten verbunden (je nach Dosierung ca. 50-100Euro/Monat).

\section{Verdauungsstörungen beim Hund}

Bei Hunden mit rezidivierender Diarrhoe, die mehrfach mit Antibiose und Cortison behandelt wurden, hat sich neben einer konsequenten Futterumstellung die Unterstützung der Darmschleimhautregeneration mit einer Mischung aus Hericium und Pleurotus bewährt. Grundsätzlich muss wegen der starken entgiftenden Wirkung der Pilze mit einer sehr geringen Dosierung begonnen werden (ca. 1/4-1/2 Kapsel/Tag), da es sonst zu weiterer Diarrhoe als Ausscheidungsreaktion kommt. Die Dosis wird alle paar Tage auf bis zu 1-

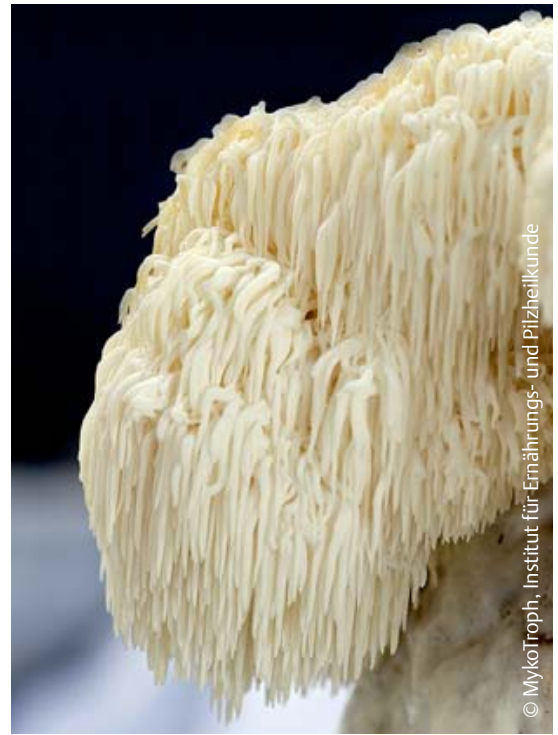

Abb. 2 Hericium - Igelstachelbart.

2 Kapseln gesteigert (abhängig von der Größe des Hundes). Die Regeneration dauert lange, deshalb ist die Dauer der Gabe in den meisten Fällen für etwa drei Monate zu planen. Die meisten Hunde nehmen die Pilze gut auf - manchmal dauert die Gewöhnung etwas länger. Die Gabe an Katzen ist oft äußerst schwierig, gerade Katzen mit Verdauungsstörungen lehnen die Pilze samt Futter oft komplett ab.

\section{Verwendete Pilze Cordyceps (Chinesischer Raupenpilz)}

Cordyceps sinensis hat sein natürliches Vorkommen im tibetischen Hochland auf 3000 bis 5000 Metern. Er wächst auf einer Raupenart und treibt aus dieser seinen Fruchtkörper. Aufgrund dieses Umstandes ist der Anbau in Deutschland verboten, d.h. Cordyceps-Präparate sind immer importiert. Aus Sicht der TCM unterstützt Cordyceps die Niere, er speichert Energie, verleiht Kraft und Ausdauer, und gilt daher als Wurzel des Lebens. In Studien wurde nachgewiesen, dass der Leberstoffwechsel verbessert und der Gallefluss angeregt wird. Eine Stunde nach Verabreichung kommt es zu einer dosisabhängigen Erhöhung der Kortikosteroide. Die Aktivität der natürlichen Killerzellen wird erhöht. Über ein Stimulieren der Peyerschen Platten wird das Immunsystem angeregt. Der Inhaltsstoff Cordycepin hat eine antibiotische Wirkung gegen Clostridium perfringens und Clostridium paraputrificum [2].

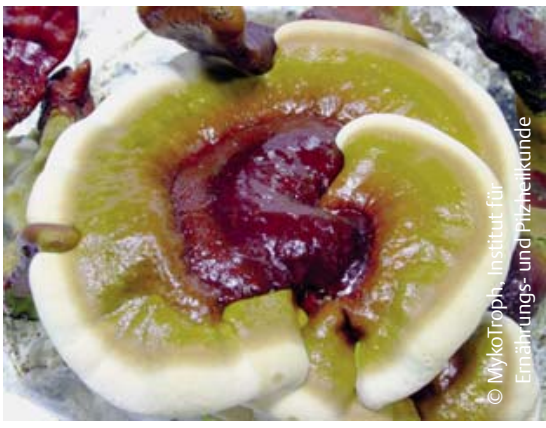

Abb. 3 Reishi - glänzender Lackporling.

\section{Hericium (Igelstachelbart)}

Hericium (Hericium erinaceus) hat sein Hauptanwendungsgebiet im Aufbau der Magen- und Darmschleimhaut und ist somit angezeigt bei Futtermittelunverträglichkeiten, Allergien, Hauterkrankungen und allgemeiner Immunschwäche. In Studien wurde gezeigt, dass die enthaltenen Polysaccharide eine antitumorale Wirkung haben und die Regeneration peripherer Nerven gefördert wird [2].

\section{Maitake (Klapperschwamm)}

Maitake (Grifola frondosa) ist ein sehr seltener Pilz, der in Asien am Stammgrund von Eichen und Edelkastanien wächst. Er hat günstigen Einfluss auf die Blutfettwerte und den Blutzuckerspiegel. Er stärkt die zelluläre Abwehr. Maitake aktiviert Osteoblasten [2].

\section{Pleurotus (Austernpilz)}

Pleurotus ostreatus wächst an Ästen und Stümpfen von Laubgehölzen. Er ist reich an Vitaminen des B-Komplexes, Vitamin C und Calciferol. Enterokokken, Laktobazillen und Bifidobakterien werden im Wachstum unterstützt, weshalb er zusammen mit Hericium eine gute Kombination zur Förderung der Darmgesundheit bietet [2].

\section{Polyporus (Eichhase)}

Polyporus umbellatus ist vorwiegend in Asien heimisch und wächst auf Stümpfen von Eichen und Buchen. Er hat einen starken Bezug zum Lymphsystem [2].

\section{Reishi (Glänzender Lackporling, Ling Zhi)}

Reishi (Ganoderma lucidum) ist sowohl in Asien als auch in Europa heimisch. Er wächst an feuchten Standorten auf Stäm- 
men und Stümpfen von Laubgehölzen. Er hat eine stark entzündungshemmende und entgiftende Wirkung. Er ist einer der wichtigsten Pilze bei der Behandlung von Allergien, da die enthaltenen Triterpene die Histaminausschüttung hemmen. Reishi hat einen starken Bezug zu Leber und Lunge und eine stressreduzierende Wirkung. Die Wirkungen des Reishi und seiner Inhaltsstoffe sind in zahlreichen Studien gezeigt worden [2].

\section{Fazit}

Insgesamt stellen die Heilpilze eine potente Therapieform dar, die auch ohne weiteres Wissen über TCM zu erlernen ist. Entscheidend für den Behandlungserfolg ist die Qualität des verwendeten Pilzpräparates und die individuelle Verschreibung.

\section{(1) Summary}

\section{Case Studies On the Application of Medicinal Mushrooms in Veterinary Medicine}

In this article, mycotherapy is presented as the possibility of individualized holistic therapy in veterinary medicine. Examples of the medicinal fungi used and their mode of action are briefly presented.

\section{Key words}

medicinal mushroom - mycotherapy - holistic therapy

Diteratur

[1] Beckstett A. Researchers Identify EOTRH Risk Factors. The Horse Magazine Editor, Article 33230; 2014. Online im Internet: http://www. thehorse.com/print-article/33230

[2] Bianchi I. Moderne Mykotherapie - Der Gebrauch der Pilze in der Medizin. Niederdorfelden: Alpha One AG; 2009

[3] Hüls I, Bienert Q, Staszyk C. Equine odontoclastic tooth resorption and hypercementosis (EOTRH): Röntgenologische und makroskopisch-anatomische Befunde. Kongress der Internationalen Gesellschaft zur Funktionsverbesserung der Pferdezähne e.V. 2012

[4] Schmaus F, Hrsg. Heilen mit Pilzen - Das große Handbuch der Mykotherapie. 1. Aufl. Limeshain: MykoTroph; 2012
[5] Simhofer H, Koweika M. Vorläufige Ergebnisse einer Blindstudie über den Effekt von „Equident" bei EOTRH. CME Horses GmbH 2012. Online im Internet: http://www.better4horses. com

[6] Staszyk C, Bienert A, Kreutzer R et al. Equine odontoclastic tooth resorption and hypercementosis. The Veterinry Journal 2008; 178 : 372-379

[7] Sykora S, Pieber K, Simhofer H et. al. Isolation of Treponema and Tannerella sp from equine odontoclastic tooth resorption an hypercementosis realted periodontal diseases. Equine Vet J 2014; 46: 358-363

[8] Vanselow R. Giftige Gräser auf Pferdeweiden. 3. Aufl. Hohenwarsleben: Westarp Wissenschaften-Verlagsgesellschaft mbH; 2011

\section{Online zu finden unter}

https://doi.org/10.1055/s-0043-113881
Dr. med. vet. Svenja Thiede

Fachtierärztin für Mikrobiologie, Tierärztin für Homöopathie

Duvenstedter Berg 47

22397 Hamburg

E-Mail: info@homoeopathiede.de

1990-96 Studium Tiermedizin an der TiHo Hannover; 1996-98 Aufbaustudium Mikrobiologie und Graduiertenkolleg Zell- und Molekularbiologie TiHo Hannover; Dez. 1998 Promotion TiHo Hannover; 1999-2004 Wissenschaftliche Assistentin in verschiedenen mikrobiologischen Labors in Hannover, Berlin und Hamburg (Forschung, Diagnostik); 2001 Fachtierärztin für Mikrobiologie; seit Juni 2005 Weiterbildung Homöopathie und Vertretung in einer Gemischtpraxis in Hamburg (Kleintiere, Pferde); seit 2007 niedergelassen mit eigener Fahrpraxis in Hamburg (Homöopathie Kleintiere, Pferde); seit 2008 VeterinärmedizinUnterricht an der Berufsschule für TFA in Hamburg; 2013 Zusatzbezeichnung Homöopathie, Dozentin bei Aude Sapere 